\title{
PRESENCIA DE RADIONUCLEIDOS EN ARCILLAS DEL SISTEMA HIDROTERMAL DEL VOLCÁN TURRIALBA, COSTA RICA
}

\author{
J.R. García-Vindas ${ }^{1 *} \&$ Esteban Gazel $^{1,2}$ \\ ${ }^{1}$ Sección de Física Nuclear Aplicada, Escuela de Física, Universidad de Costa Rica \\ *Autor para contacto: jrgarcia@ cariari.ucr.ac.cr \\ ${ }^{2}$ Escuela Centroamericana de Geología, Universidad de Costa Rica, \\ Apdo. 214-2060, Costa Rica
}

(Recibido 31/10/02; aceptado 22/04/03)

\begin{abstract}
The first results obtained about the presence of radionucleids of terrigenous origin in the hydrothermal system of Turrialba Volcano are shown in this paper. Several samples were taken in the central and southwest craters, and analyzed by gamma-ray spectrometry, with a Hyperpure Germanium detector. The values of the activities of the natural radioactive isotopes are shown in Bq/kg. A disequilibrium in the series of U-238 was found, which is explained by two different mechanisms: one convective and the other by Rn-222 enrichment. An increase of the activity of all isotopes is observed between September 2001 and May 2002. Because the low possibilities that this can be produced by the water runoff, it it proposed that such rise in the concentration of the elements can be explained by their presence in the water vapour and other volcanic gases.
\end{abstract}

Keywords: Radionucleids, clays, hydrothermal system, Turrialba volcano, volcanic gases.

RESUMEN: Los primeros resultados de un estudio sobre la presencia de algunos radionucleidos de origen terrígeno, son presentados para el sistema hidrotermal del volcán Turrialba. Se tomaron muestras de arcillas en varios puntos de los cráteres central y suroeste y se analizaron mediante espectrometría gamma de bajo nivel, usando un detector de germanio hiperpuro. Se reportan los valores de las actividades en $\mathrm{Bq} / \mathrm{kg}$, de los isótopos radiactivos naturales detectados. Se encontró un desequilibrio en la serie del U-238, el cual es explicado por dos mecanismos diferentes: uno de índole convectivo y el otro por enriquecimiento de Rn-222. Se observa un aumento en la actividad de todos los isótopos entre setiembre del 2001 y mayo del 2002. Dada la poca probabilidad de que este aumento se deba a la escorrentía superficial, se propone que el aumento de la concentración de estos elementos puede ser ocasionado por su llegada en la fase gaseosa, suspendidos en el vapor de agua y otros gases volcánicos.

Palabras clave: Radionucleidos, arcillas, sistema hidrotermal, volcán Turrialba, gases volcánicos.

\section{INTRODUCCIÓN}

El análisis de isótopos radiactivos naturales en sistemas hidrotermales volcánicos ha sido el objeto de algunos estudios recientes en varios volcanes del mundo. Estos isótopos radiactivos pueden presentarse en forma gaseosa, como el $\mathrm{Rn}-222$, o en forma de aerosol, como el Po-218 o $\mathrm{Pb}-210$. Estos últimos pueden ser arrastrados desde el magma, por algunos gases o alcanzar la 
superficie, suspendidos en el vapor de agua hasta llegar a la pluma exhalativa (Le Cloarec $\&$ Pennisi, 2001). Algunos de ellos han mostrado ser buenos indicadores de la actividad volcánica pre-eruptiva, tal es el caso del Rn-222, que en algunos casos ha sido un posible precursor de eventos mayores (Seidel et al., 1988; Connor et al., 1996; García-Vindas et al., 2002). Muchos de estos radionucleidos se han encontrado en muestras de coladas de lava (Gill et al., 1985; Segovia, 1990) y en algunos casos se ha mostrado la existencia de un posible enriquecimiento del magma con uno de estos antes de aflorar a la superficie, como es el caso del Ra-226 (Wordel et al., 1994). El estudio de la presencia de estos radioisótopos nos ayuda a entender la naturaleza de algunos procesos pre-eruptivos y en algunos casos, conocer la génesis del material que aflora en la superficie (Reagan et al., 1994).

En el presente trabajo se han analizado los radionucleidos presentes en un conjunto de muestras de arcillas del sistema hidrotermal del volcán Turrialba, como un primer ensayo o estudio de los elementos presentes.

\section{ASPECTOS GEOLÓGICOS}

La Cordillera Volcánica Central es parte del arco magmático cuaternario centroamericano, que se extiende desde la frontera MéxicoGuatemala hasta el complejo Irazú-Turrialba en Costa Rica. Este arco es el producto de la subducción de la placa del Coco bajo la del Caribe y sus características varían de acuerdo con las propiedades estructurales y tectónicas de la región (Carr et al., 1989). Esta cordillera presenta una longitud de $80 \mathrm{~km}$ y está conformada por cinco macizos volcánicos complejos, del NW al SE: Platanar - Porvenir, Poás, Barva, Irazú y Turrialba. Todos presentan una composición mineralógica muy similar: andesitas basálticas y andesitas (Kussmaul et al., 1994).

El volcán Turrialba $\left(10^{\circ} 02^{`} \mathrm{~N}-83^{\circ} 46^{`} \mathrm{~W}\right)$ es el más oriental de la Cordillera Volcánica Central de Costa Rica (Fig. 1). Su elevación

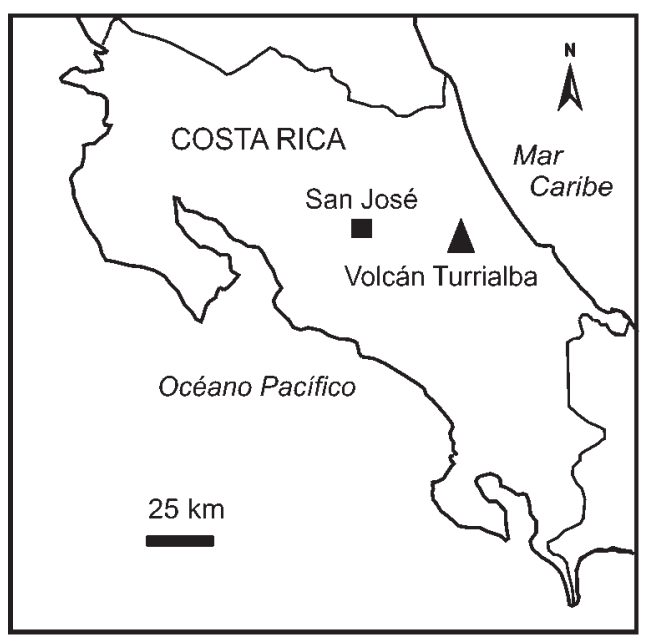

Fig. 1: Ubicación de la zona de estudio.

máxima sobre el nivel del mar es de unos 3340 $\mathrm{m}$, el edificio volcánico presenta una altura de $1900 \mathrm{~m}$ y un volumen aproximado de $290 \mathrm{~km}^{3}$ (Carr, 1984). Este volcán se desarrolló sobre la Cuenca Sedimentaria de Limón Sur y estructuras volcánicas pliocénicas alcalinas y andesíticas (Tournon, 1984; Soto, 1988; Alvarado, 2000). La secuencia completa está cortada por fracturas tensionales de dirección NE, responsables de la instalación del conjunto del volcán Turrialba (Soto, 1988).

La actividad volcánica del Turrialba se estima que se inició aproximadamente hace $75 \mathrm{ka}$ (Reagan \& Gill, 1989). Los depósitos predominantes, corresponden a piroclastos de caída, flujos y oleadas piroclásticas y avalanchas. Las fuentes mantélicas son calcoalcalinas y de intraplaca, generando una mezcla de magmas (Reagan \& Gill, 1989). Soto (1988) menciona que la mayoría de los eventos explosivos corresponden con erupciones estrombolianas, aunque también se presentan depósitos freáticos y freatomagmáticos, y se han observado dos eventos plinianos. La última actividad explosiva de este volcán sucedió entre 18641866. Posteriormente a esta actividad se ha presentado actividad solfatárica en los cráteres central y suroeste (Alvarado, 2000). 


\section{METODOLOGÍA}

\section{Recolección y preparación de las muestras}

Las muestras de arcillas se recolectaron durante los meses de setiembre y noviembre del año 2001 y mayo del 2002, en fumarolas activas, con temperaturas que varían desde $\operatorname{los} 86^{\circ} \mathrm{C}$ a los $90^{\circ} \mathrm{C}$. Estas fumarolas se localizan en el norte del cráter central y en el borde norte del cráter suroeste (Fig. 2). El material arcilloso presenta un color generalmente blanco, se encuentra a una temperatura aproximada a los $80^{\circ} \mathrm{C}$ y es corrosivo (oxida espontáneamente el metal). Las rocas que están siendo alteradas en los lugares de muestreo corresponden con material piroclástico (cenizas, lapilli y bombas) relativamente fresco (posiblemente el arrojado en las últimas erupciones). En ocasiones dentro de las arcillas hidrotermales se han encontrado mineralizaciones de azufre nativo e inclusiones de rocas aún no alteradas o en procesos de alteración.

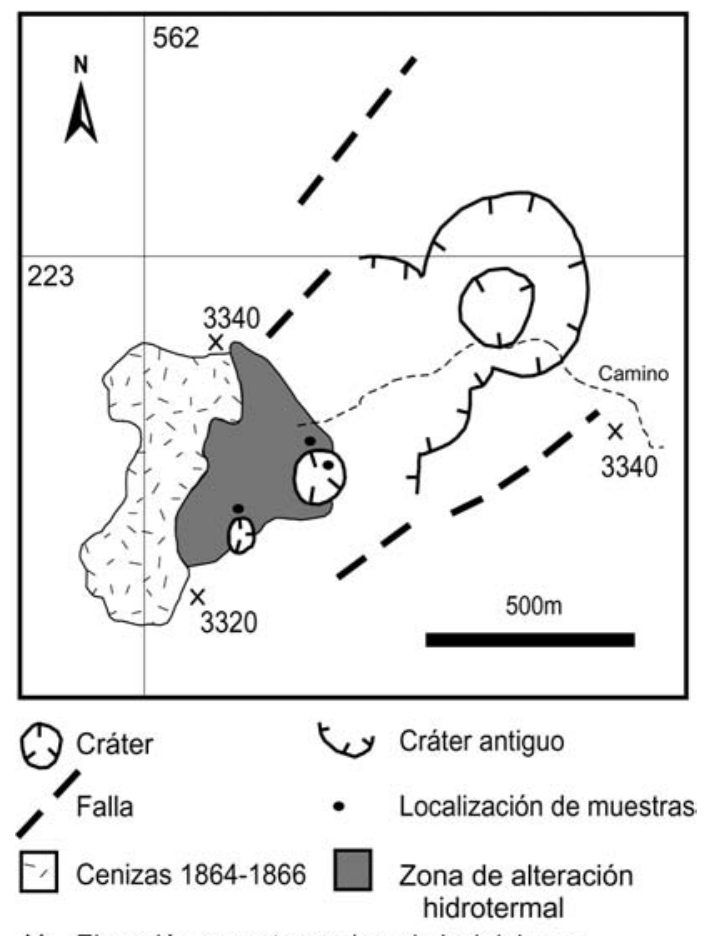

$\times$ Elevación en metros sobre el nivel del mar

Fig. 2: Esquema de los cráteres del volcán Turrialba y ubicación de los sitios de recolección de las muestras (Modificado de Soto, 1994).
Se recolectó alrededor de un kilogramo de muestra representativa en cada solfatara seleccionada. La misma se toma, aproximadamente por debajo de los $5 \mathrm{~cm}$ de profundidad, removiendo por completo el material de la superficie. Esto permite eliminar la posibilidad de incluir en la muestra aquellos radioisótopos hidrosolubles que hayan podido ser transportados por la escorrentía superficial. Por otro lado, los sitios de muestreo escogidos se encuentran por lo general sobre pequeños montículos, por lo que la posibilidad de que estos se encuentren contaminados con material transportado por escorrentía es escasa. Asimismo, se recolectaron algunas muestras retrabajadas o expuestas al medio, con el fin de observar la existencia de transporte de radionucleidos por medio de aguas superficiales. Las muestras se trasladaron al laboratorio de Física Nuclear Aplicada de la Universidad de Costa Rica, se secaron, molieron y tamizaron con una malla de $500 \mu \mathrm{m}$. Para el secado se utilizó un horno de convección a una temperatura de $60^{\circ} \mathrm{C}$ y para el molido, un mortero de ágata. Se tomó un volumen de muestra de aproximadamente $500 \mathrm{~cm}^{3}$ y se depositó en un recipiente tipo Marinelli.

\section{Análisis de las muestras}

Las muestras se analizaron por espectrometría gamma de bajo nivel, la cual consiste en la obtención del espectro de emisión de la radiación gamma emitida por los isótopos radiactivos utilizando un detector de cristal de germanio hiperpuro (Intertechnique EGPC 20, 5,1 cm de diámetro, $21,9 \%$ de eficiencia relativa y una resolución de $1,88 \mathrm{keV}$ ), acoplado a un amplificador que a su vez se conecta a un convertidor analógico digital y éste a un analizador multicanal con 8096 canales. Este último, se acopla a una computadora con el software para el análisis de espectros. El multicanal clasifica cada elemento por energías, para luego desplegar en la pantalla de una computadora el espectro completo de energías. El detector está blindado con paredes de plomo de $8 \mathrm{~cm}$ de espesor para disminuir la contribución de la radiación del ambiente. La parte interior de las paredes de plomo se recubrió con 0,5 mm de PMMA y una capa de cobre de $1 \mathrm{~mm}$ para blindar el detector de 
la fluorescencia de rayos X. Los resultados obtenidos en $\mathrm{Bq} / \mathrm{kg}$ se pueden expresar en ppm haciendo uso de las ecuaciones de desintegración (Eisenbud \& Gesell, 1997). Los procedimientos usados para calcular la calibración en energía y la eficiencia, así como el análisis de los picos se describen en Loría \& Mora (1993).

\section{RESULTADOS}

En el Cuadro 1 se presenta la localidad y descripción del tipo de recolección de cada muestra. La muestra 1 se tomó en setiembre del 2001, las muestras 2, 3, 4 y 5 durante noviembre del mismo año. Por último, las muestras 6,7 y 8 se recolectaron en mayo del 2002. Todas las muestras, con excepción de la 3 y la 4 se tomaron directamente en las fumarolas. La muestra 2 fue tomada en otra fumarola diferente a la escogida para las muestras 1, 5, 6, 7 y 8, sin embargo, este sitio no se siguió muestreando. Las muestras 3 y 4 se recolectaron en el fondo del cráter central y corresponden con material retrabajado (erosionado, transportado y depositado por la escorrentía superficial). La muestra 7 corresponde a material superficial, expuesto a las condiciones del medio y que no ha sido retrabajado.

Los Cuadros 2 y 3 muestran las actividades, en $\mathrm{Bq} / \mathrm{kg}$, de los principales radionucleidos presentes en cada muestra. En el Cuadro 2, la columna 2 corresponde con el K-40, elemento abundante en este tipo de muestras. Los elementos presentes en las columnas 3,4 y 5 forman parte de la cadena de desintegración del Th-232. La última columna de este cuadro presenta la razón entre el Pb-212 y el Bi-212. En el cuadro 3, los elementos de las columnas 2, 3, 4 y 5 forman parte de la cadena del U-238, mientras que en las siguientes dos columnas se presentan dos radionucleidos pertenecientes a la cadena del U-235. La última columna presenta la razón entre el $\mathrm{Pb}$ 214 y el Bi-214.

\section{DISCUSIÓN Y CONCLUSIONES}

Del Cuadro 2 se desprende que los isótopos de la serie del Th-232, para cada muestra, se encuentran en equilibrio, pues la razón $\mathrm{Pb}$ 212/Bi-212 es cercana a la unidad. Lo mismo ocurre para los isótopos de la serie del U-238,

Cuadro 1

Descripción y ubicación de las muestras recolectadas

\begin{tabular}{|c|c|c|}
\hline Muestra & Descripción & Localidad \\
\hline 1 & $\begin{array}{l}\text { Arcilla hidrotermal recolectada } \\
\text { directamente en la solfatara. }\end{array}$ & Solfatara al norte del cráter central \\
\hline 2 & Ídem a la muestra 1 & Solfatara al noreste del cráter central \\
\hline 3 & $\begin{array}{l}\text { Arcilla retrabajada y } \\
\text { redepositada por la escorrentía } \\
\text { superficial }\end{array}$ & Fondo del cráter central \\
\hline 4 & Ídem a la muestra 3 & Fondo del cráter central \\
\hline 5 & $\begin{array}{l}\text { Arcilla hidrotermal recolectada } \\
\text { directamente en la solfatara. }\end{array}$ & Solfatara al norte del cráter central \\
\hline 6 & Ídem a la muestra 5 & Solfatara al norte del cráter central \\
\hline 7 & $\begin{array}{l}\text { Arcilla hidrotermal recolectada } \\
\text { directamente en la solfatara, } \\
\text { pero tomada a } 0 \mathrm{~cm} \text {, expuesta a } \\
\text { las condiciones del medio }\end{array}$ & Solfatara al norte del cráter central \\
\hline 8 & $\begin{array}{l}\text { Arcilla hidrotermal recolectada } \\
\text { directamente en la solfatara }\end{array}$ & Solfatara al norte del cráter oeste \\
\hline
\end{tabular}


mostrados en el Cuadro 3, en la que se observa equilibrio entre el $\mathrm{Bi}-214$ y $\mathrm{Pb}-214$, sin embargo, el equilibrio se rompe con el Ra-226 en cada muestra. Si se observan las muestras $1,2,5$ y 6 , las cuales corresponden con material no expuesto a las condiciones del medio, recolectado en el cráter central, las actividades de Ra-226, con excepción de la muestra 2 (esta muestra corresponde a otra fumarola), se encuentran por debajo de las concentraciones correspondientes de $\mathrm{Pb}-214$ y Bi-214 para cada muestra. Este desequilibrio puede ser explicado por dos mecanismos diferentes. El primero puede ser la pérdida de este elemento a causa del agua ya que, aunque el material no se encuentra directamente en la superficie, pudo haber sido desplazado por el agua que se infiltra en el lugar. La otra razón podría ser que el material recolectado haya atrapado Rn-222 antes de aflorar a la superficie y enfriarse, por lo que las actividades de sus descendientes (Pb-214 y Bi-214) se ven aumentadas. Esto último ya ha sido puesto en evidencia antes (de la Cruz-Reyna et al., 1991). Por otro lado, al observar los resultados de la muestra 7, la cual corresponde con material expuesto al ambiente, se induce que el Ra-226 ha sido probablemente removido, en parte, de la superficie, ya que el desequilibrio con sus descendientes es mayor.

La muestra 8, la cual fue recolectada en el cráter suroeste, presenta el mismo comportamiento que las muestras anteriormente mencionadas. Otro aspecto que se debe recalcar de esta muestra es el hecho de que aparece el isótopo Pa$234 \mathrm{~m}$ en cantidad suficiente como para ser detectado, lo cual indica que hay presencia de U-238 en la muestra. Si asumimos que hay equilibrio

Cuadro 2

Actividad en $\mathrm{Bq} / \mathrm{kg}$ de algunos de los radioisótopos analizados

\begin{tabular}{cccccc}
\hline $\mathrm{N}^{\circ}$ & ${ }^{40} \mathrm{~K}$ & ${ }^{212} \mathrm{Bi}$ & ${ }^{212} \mathrm{~Pb}$ & ${ }^{228} \mathrm{Ac}$ & ${ }^{212} \mathrm{~Pb} /{ }^{212} \mathrm{Bi}$ \\
\hline 1 & 384,40 & 21,76 & 29,54 & 28,96 & 1,4 \\
2 & $3,08 \mathrm{x}$ & n.d. & 1,51 & $1,78 \mathrm{x}$ & - \\
3 & 285,84 & $13,83 \mathrm{x}$ & 17,98 & 20,98 & 1,3 \\
4 & 354,73 & 13,53 & 19,89 & 20,16 & 1,5 \\
5 & 331,11 & 21,40 & 30,95 & 28,95 & 1,4 \\
6 & 209,64 & 39,14 & 52,48 & 62,07 & 1,3 \\
7 & 432,83 & 39,64 & 49,67 & 54,98 & 1,3 \\
8 & 365,12 & 50,16 & 66,93 & 73,37 & 1,3 \\
\hline
\end{tabular}

Los valores cercanos al valor mínimo detectable por el instrumento se encuentran marcados con "x" n.d.= No se detectó el isótopo, o el valor está por debajo del mínimo detectable

Cuadro 3

Actividad en $\mathrm{Bq} / \mathrm{kg}$ de algunos de los radioisótopos analizados

\begin{tabular}{crrccccc}
\hline $\mathrm{N}^{\circ}$ & ${ }^{214} \mathrm{Bi}$ & ${ }^{214} \mathrm{~Pb}$ & ${ }^{226} \mathrm{Ra}$ & ${ }^{234 \mathrm{~m} \mathrm{~Pa}}$ & ${ }^{227} \mathrm{Th}$ & ${ }^{235} \mathrm{U}$ & ${ }^{214} \mathrm{~Pb} /{ }^{14} \mathrm{Bi}$ \\
\hline 1 & 45,05 & 37,15 & 29,49 & n.d. & 2,56 & $1,49 \mathrm{x}$ & 0,8 \\
2 & 34,04 & 37,47 & 40,79 & n.d. & n.d. & 2,48 & 1,1 \\
3 & 29,79 & 26,58 & 10,76 & $8,35 \mathrm{x}$ & 2,80 & 2,15 & 0,9 \\
4 & 31,46 & 29,29 & $5,74 \mathrm{x}$ & $6,39 \mathrm{x}$ & n.d. & 2,38 & 0,9 \\
5 & 37,43 & 34,23 & 12,40 & n.d. & 3,54 & 2,12 & 0,9 \\
6 & 125,15 & 109,03 & 85,06 & n.d. & 12,11 & 3,24 & 0,9 \\
7 & 93,67 & 78,69 & 43,66 & n.d. & 7,77 & 3,60 & 0,8 \\
8 & 89,91 & 75,13 & 70,42 & 134,66 & 4,53 & 4,58 & 0,8 \\
\hline
\end{tabular}

Los valores cercanos al valor mínimo detectable por el instrumento se encuentran marcados con " $\mathrm{x}$ " n.d.= No se detectó el isótopo, o el valor está por debajo del mínimo detectable 
secular entre ambos elementos, la cantidad de uranio presente en esta muestra es de 11 ppm. Este valor sobrepasa lo reportado por otras fuentes para este tipo de material (Larsen \& Phair, 1959; Eisenbud \& Gesell, 1997).

Otro aspecto importante que se debe recalcar es el hecho de que las muestras recolectadas durante el año 2002 (6, 7 y 8) presentan una mayor actividad en los elementos de las tres series, que aquellas que fueron tomadas durante el 2001. Considerando que el sitio de muestreo es el mismo, en lo que respecta al cráter central, se induce que este aumento en la actividad de todos los elementos puede deberse a un aumento en la concentración de estos en la fase gaseosa de las solfataras, pues la única manera de que estos lleguen a la muestra es suspendidos en el vapor de agua o en los gases que llegan a la superficie, ya que como se mencionó anteriormente, la morfología del terreno impide que la escorrentía proveniente de otros sitios llegue al lugar de muestreo. Un indicio de que estos elementos podrían ser transportados por el vapor u otros gases hasta contaminar la muestra, es el hecho de que en la muestra 7, la cual contiene material expuesto, presenta también un aumento en la actividad de todos sus elementos con excepción del Ra-226, el cual es muy soluble en agua y se lava fácilmente con la escorrentía, por lo que su concentración puede verse disminuida.

En conclusión, un posible aumento de la concentración de estos radionucleidos en la fase gaseosa de las solfataras pudo haber tenido lugar entre setiembre del 2001 y mayo del 2002. Por otro lado, se induce también que el desequilibrio en la serie del U-238 pudo deberse, en parte, a un enriquecimiento del material con Rn-222 antes de aflorar a la superficie y posteriormente enfriarse. Durante el proceso ulterior de solidificación del material, el Rn-222 pudo haber quedado atrapado y por decaimiento, aumentar la concentración de sus descendientes.

Es necesario realizar otro tipo de estudios para complementar este trabajo, como por ejemplo, realizar un análisis de los condensados en las solfataras por medio de espectrometría alfa, con el fin de determinar si hay Ra-226 y U-238 presentes en el vapor de agua. Esto podría probar la hipótesis que se plantea en el presente trabajo, concerniente al transporte de estos radioisótopos por el vapor de agua, lo cual, de ser verdadero, reflejaría sin duda que podría estarse produciendo un cambio en los procesos internos del volcán.

\section{AGRADECIMIENTOS}

Los autores desean expresar su agradecimiento a la Vicerrectoría de Investigación de la Universidad de Costa Rica (Proyecto Factores Multiparamétricos Radiactivos en los Volcanes de Costa Rica, \# 112-98-364) y a la Agencia Internacional de Energía Atómica (Proyecto Impacto Ambiental y Sanitario de las Emanaciones Volcánicas en Costa Rica, \# COS7/002) por su apoyo y colaboración. Asimismo manifestarle un especial agradecimiento al Sr. Mauricio Badilla, investigador del Laboratorio de Física Nuclear Aplicada, por su colaboración en el análisis de las muestras y al Dr. Siegfried Kussmaul por sus oportunos comentarios.

\section{REFERENCIAS}

ALVARADO, G.E., 2000: Los volcanes de Costa Rica: geología, historia y riqueza natural [2a ed.]. - 269 págs. Ed. UNED, San José.

CARR, M.J., 1984: Symmetrical and segmented variation of physical and geochemical characteristics of the Central American volcanic front. - J. Volcanol. Geotherm. Res. 20: 231-252

CARR, M.J., FEIGENSON, M.D. \& BENNETT, E.A., 1989: Incompatible element and isotopic evidence for tectonic control of source mixing and melt extraction along the Central American arc. - Contrib. Mineral. Petrol. 105: 369-380

CONNOR, C.; HILL, B., LAFEMINA, P., NAVARRO, M. \& CONWAY, M., 1996: Soil Rn-222 pulse during the initial phase of the June-August 1995 eruption of Cerro Negro Nicaragua. - J. Volcanol. Geotherm. Res. 73: 119-127.

DE LA CRUZ-REYNA, S.; ARMIENTA-HERNÁNDEZ, M. A. \& SEGOVIA, N., 1991: Some isotopic and geochemical anomalies observed in Mexico prior to large scale earthquakes and volcanic eruptions. - En: 
Isotopic and geochemical precursors of earthquakes and volcanic eruptions.- Proceedings of an Advisory Group Meeting held in Vienna, 9-12 September: 63-87.

EISENBUD, M. \& GESELL, T., 1997: Environmental radioactivity from natural, industrial and military sources [4a ed.].- 656 págs. Academic Press, San Diego, California.

GARCÍA-VINDAS, J.R., FERNÁNDEZ, E., DUARTE, E. \& MALAVASSI, E., 2002: Radon concentration anomalies as possible precursors to eruptions of the Arenal Volcano. - Geofís. Int. 41(3B).

GILL, J., WILLIAMS, R. \& BRULAND, K., 1985: Eruption of basalt and andesite lava degasses ${ }^{222} \mathrm{Rn}$ and ${ }^{210} \mathrm{Po}$. - Geophys. Res. Lett. 12(1): 17-20.

KUSSMAUL, S., TOURNON, J., \& ALVARADO, G., 1994: Evolution of the Neogene to Quaternary igneous rocks of Costa Rica. - Profil, 7: 97-123.

LARSEN, E.S. \& PHAIR G., 1959: The distribution of uranium and thorium in igneous rocks - En: Nuclear Geology, 414 págs. Ed. Wiley, New York.

LE CLOAREC, M.F. \& PENNISI M., 2001: Radionuclides and sulfur content in Mount Etna plume in 19831995: new constraints on the magma feeding. - J. Volcanol. Geotherm. Res. 108: 141-155.

LORÍA, L.G. \& MORA, P., 1993: Specific activity and derived intervention levels for Cesium-137 in Costa Rican export goods: tuna fish, coffee and powdered milk. - Radiation Measurements, 23: 731-736.

REAGAN, M.K., \& GILL, J.B., 1989: Coexisting calcalkaline and high-Niobium basalts from Turrialba volcano, Costa Rica: Implications for residual titanites in arc magma sources. - J. Geophys. Res. 94: 4619-4633
REAGAN, M., MORRIS, J., HERRSTROM, E., \& MURRELL, T., 1994: Uranium series and beryllium isotope evidence for an extended history of subduction modification of the mantle below Nicaragua. - Geochim. Cosmochim. Acta, 58(19): 41994212.

SEGOVIA, N., 1990: Radioactivity outburst from geophysical events. High levels of natural radiation. - Proceedings of an international conference, Ramsar, 3-7 November: $317-324$.

SEIDEL, J.L.; MONNIN, M.; BONEVILLE, A.; DELORME, H.; LÉNAT, J.F. \& SEGOVIA, N., 1988: Radon in soil measurements related to the activity of the Piton de la Fournaise volcano, La Réunion Island, France. - Nucl. Tracks. Radiat. Meas. 15(1-4): 567570.

SOTO, G.J., 1988: Estructuras volcano-tectónicas del Volcán Turrialba, Costa Rica, América Central. - V Congreso Geol. Chileno, 8-12 de agosto de 1988, Santiago, Tomo III: I163-I175.

SOTO, G.J., 1994: Vulcanología Física.- En: P. DENYER \& S. KUSSMAUL (comp.): Atlas geológico Gran Área Metropolitana. - Ed. Tecnológica de Costa Rica, Cartago: 249-261.

TOURNON, J.,1984: Magmatismes du Mesozoique à l'actuel en Amérique Centrale: 1'exemple de Costa Rica, des ophiolites aux andésites. - 335 págs., Univ. Pierre et Marie Curie [Tesis doctoral].

WORDEL, R.; MOUCHEL, D.; SOLÉ, V.A.; HOOGEWERFF, J. \& HERTOGEN, J., 1994: Investigation of the natural radioactivity of volcanic rock samples using a low background gamma-ray spectrometer. Nuclear Instruments and Methods in Physics Research, A(339): 322-328. 